\title{
Impulsivity and Inhibitory Control in Normal Development and Childhood Psychopathology
}

\author{
Russell Schachar \\ Department of Psychiatry \\ The Hospital for Sick Children \\ Toronto, Ontario, Canada
}

\author{
Gordon D. Logan \\ University of lllinois
}

\begin{abstract}
Two experiments investigated the development and pathology of inhibitory control in children. Inhibitory control was investigated with the stop-signal paradigm, which is based on a formal theory of inhibition and directly measures the mechanism of inhibition. The ability to inhibit developed little after Grade 2, but subjects with attention deficit disorder with hyperactivity (ADDH) showed deficient inhibitory control. Their deficient inhibitory control was attributable to the subgroup of ADDH subjects with pervasive hyperactivity who had a more severe inhibitory deficit than did the situational hyperactive subgroup, the normal group, and the pathological controls. These studies reflect the utility of the stop-signal paradigm as a measure of inhibitory control.
\end{abstract}

The concept of inhibitory control is central in theories of child development and in the definition and explanation of psychopathological disorders of childhood-in particular, of attention deficit disorder with hyperactivity (ADDH; Douglas, 1983; Kogan, 1983; Milich \& Kramer, 1984). Inhibitory control is one of several processes that perform the executive functions of the cognitive system. These functions determine how various mental processes (e.g., encoding, recognition, retrieval) will work together in the performance of a task. Children need executive control to choose, construct, execute, and maintain optimal strategies for performing a task, as well as to inhibit strategies that become inappropriate when goals or task demands change or errors occur (Logan, 1985). Deficient inhibitory control is revealed by impulsive behaviors such as responding before the task is understood, answering before sufficient information is available, allowing attention to be captured by irrelevant stimuli (i.e, distractibility), or failing to correct obviously inappropriate responses. Poorly developed inhibitory control might account for impulsive behaviors of younger children and of children with various types of psychopathology.

Despite the importance of inhibitory control in theories of child development and psychopathology, no widely accepted

We would like to acknowledge the contributions of Diane Chajczyk, Pat Fulford, Haraldene Phair, and Rosemary Tannock in the conduct of this study and the support of the Ontario Mental Health, Ruth Schwartz, and Canadian Psychiatric Research Foundations. The manuscript was prepared with the assistance of the Medical Publications Department, The Hospital for Sick Children, Toronto, Ontario, Canada.

Correspondence concerning this article should be addressed to Russell Schachar, Department of Psychiatry, The Hospital for Sick Children, 555 University Avenue, Toronto, Ontario, Canada, M5G 1X8 or to Gordon D. Logan, Department of Psychology, University of Illinois, 603 East Daniel Street, Champaign, Illinois 61820. Electronic mail may be sent to g-logan @ h. psych. uiuc. or to attn @ sick kids. utoronto. ca. measure of inhibition exists. Instead, impulsivity is defined operationally (e.g., calling out in class or demonstrating an inability to plan performance on a maze task). Most frequently, impulsivity and inhibitory control are studied with laboratory tasks that involve response uncertainty, careful planning, and slow responding. The most common measure is the Matching Familiar Figures Task (MFFT; Kagan, Rosman, Day, Albert, \& Phillips, 1964). This task requires subjects to search a number of similar pictures for one that matches a criterion picture exactly. Fast and inaccurate performance on the MFFT is assumed to indicate impulsivity or lack of cognitive control over the execution of the response; the subject is believed to be unable to delay a response in the course of analyzing the stimuli and searching for the correct alternative.

Studies using the MFFT indicate that as normal children grow older, they develop longer response latencies and greater accuracy (Salkind \& Wright, 1977). In contrast, children with clinical disorders characterized by apparent impulsivity, such as hyperactive children (attention deficit disorder, ADDH), respond more quickly and make more errors (see Campbell, Douglas, \& Morgenstern, 1971). These differences are interpreted as evidence of deficient impulse control in hyperactive and younger children. Impulsive performance on the MFFT and other tasks such as the continuous performance task is a major argument for the hypothesis that deficient impulse control is one of the basic problems of hyperactive children (Douglas, 1983; Douglas \& Peters, 1979).

Performance on these tasks, however, depends on factors other than cognitive impulsivity. For example, MFFT performance varies with IQ (Milich \& Kramer, 1984); search strategy (Ault, Crawford, \& Jeffrey, 1972); metacognitive awareness of appropriateness of inhibiting the response until all variants have been compared (Brown, Bransford, Ferrara, \& Campione, 1983); or decision criteria, motivation, or attentional capacity (Kahneman, 1973). Consequently, the impulsivity that the MFFT and similar tasks reveal in younger and hyperactive 
children could be accounted for by deficits in one or several processes. Tasks like the MFFT may help distinguish between individuals or groups but do not clarify the underlying psychological processes that produce impulsivity.

In addition to difficulties in measuring impulsivity, clinical studies have been hampered by a lack of diagnostic precision. Many studies of impulsivity in hyperactive children have used samples of children exhibiting a variety of comorbid disorders or have not included pathological controls. Hyperactive children typically present with a variety of other disturbances, such as conduct and learning disorders. To determine whether hyperactivity rather than one or all of these correlated disorders is associated with cognitive impulsivity, hyperactive children must be compared with children who have one of these disorders, as well as with normal children.

In this study, we investigated the development and pathology of inhibitory control through the use of a new stop-signal paradigm that allows direct assessment of inhibitory control. In the first experiment, we focused on the development of inhibitory control in normal children as measured in the stop-signal paradigm. In the second experiment, we compared the inhibitory control of normal children with that of children who have a psychopathological condition characterized clinically by poor impulse control (ADDH). To determine whether a deficit in inhibitory control is specific to ADDH, we compared the performance of these children with that of children who have emotional, learning, and conduct disorders.

\section{The Stop-Signal Paradigm}

The stop-signal paradigm is a laboratory analog of a situation requiring inhibitory control that distinguishes stimuli that elicit impulsive behavior (primary-task stimuli) from those that inhibit it (stop-signal stimuli). Subjects engaged in a primary task (e.g., forced-choice letter discrimination) are presented with an occasional stop-signal stimulus (e.g., a tone) instructing them to inhibit their response to the primary-task stimulus. Unlike methods usually used in studies of childhood impulsivity (e.g., Kagan et al., 1964), the stop-signal paradigm is based on a theory of inhibition (Logan \& Cowan, 1984; Logan, Cowan, \& Davis, 1984) and directly measures the mechanism of inhibition. In the real world, the stimuli that elicit impulsive behavior and those that inhibit it sometimes overlap (e.g, when the deviance or inappropriateness of the response is the signal to inhibit it). We attempted to apply this paradigm only with clearly discriminable "go" and "stop" stimuli to establish the basic effects of development and psychopathology.

Childhood impulsivity may be associated with deficiency of inhibitory control in two ways: The stop-signal stimulus either may fail to trigger the stop-signal response or may trigger a response that is slower or more variable than normal (see Luce, 1986).

\section{Inhibition Functions}

In the model of Logan and Cowan (1984), response inhibition depends on a race between the primary-task processes and the stop-signal processes. If the former win, a response occurs; if the latter win, the response is inhibited. Because the finishing times of these processes are assumed to vary randomly, the outcome of the race is a matter of probability. Thus, in the model, the main dependent variable is the probability of inhibition.

The main independent variable is the interval between the stop signal and the primary-task response (i.e, the stop-signal delay). The stop-signal delay handicaps the race, biasing it in favor of one process or the other. If the signal occurs early enough (i.e., well before the primary-task stimulus), subjects will inhibit every time. If it occurs late enough (i.e, after the response to the primary task), subjects will never inhibit. Between these extremes, the probability of inhibition diminishes gradually from 1.0 to 0.0 . Plotting the probability of inhibition against stop-signal delay generates an inhibition function whose shape depends on the distribution of primary-task reaction times and the distribution of internal reaction times to the stop signal (the stop-signal reaction time, or SSRT; for a formal derivation, see Logan \& Cowan, 1984).

Differences in the efficiency of the inhibition process will affect the shape of the inhibition function. In general, the better the process, the higher and steeper the function. If the inhibition mechanism were never triggered in stop-signal trials, the probability of inhibition would be 0.0 at all delays, producing a flat, low inhibition function. If the mechanism were triggered in only some stop-signal trials, the function would be steeper and higher, but not as steep and high as a normal one. The speed of the inhibition mechanism also determines the shape of the inhibition function. A slower mechanism will lose the race against the primary-task process more often than a faster one, producing a lower probability of inhibition. Extended over all delays, it will produce a lower and flatter inhibition function. If the inhibition mechanism of younger children or children with a clinical condition affecting impulse control is less likely to be triggered, is slower, or is less consistent, their inhibition functions will be flatter and lower than those of older and normal children. ${ }^{1}$

According to the race model, the shape of the inhibition function also depends on the mean and variability of the primary-task processes. If inhibition functions are linear, variation in means will affect the intercept of the inhibition function and variation in standard deviations will affect slope. In research with adults, these effects have been small (Logan et al, 1984). However, we expect greater differences among children because response times are longer and more variable in younger and disturbed children than in older and undisturbed children (Peloquin \& Klorman, 1986).

Differences in the mean and the variability of the stopping and primary-task reaction times among groups may or may not be sufficient to account for observed intergroup differences in inhibition functions. The model of Logan and Cowan (1984)

\footnotetext{
${ }^{1}$ Variability in the speed of the inhibition process also affects the steepness of the function. According to the race model, the inhibition function can be viewed as a probability distribution: The more variable the distribution, the flatter the function. The race model asserts that the variance of the inhibition function is the sum of the variance of the SSRT and the primary-task reaction times. Thus, a deficiency that increases the variance of the SSRT will flatten the inhibition function (see Logan \& Cowan, 1984).
} 
provides methods of correcting inhibition functions for differences in stopping and primary-task reaction times that can bring disparate inhibition functions into alignment.

Differences in mean primary-task reaction time can be corrected by setting the stop-signal presentation relative to the mean primary-task reaction time, as we have in our experiments (see Setting stop-signal delay for details). If inhibition functions are not aligned by this method of correcting for differences in the mean primary-task reaction time, then group differences may be a result of differences in the stop-signal reaction time or of variability in the primary-task reaction times. In order to account for differences in stop-signal reaction time, the probability of inhibition can be plotted as a function of MRT-delay-SSRT. If the functions are still not aligned, they should be plotted as a function of ZRFT (Logan et al, 1984):

$$
\text { ZRFT }=\frac{\text { MRT }- \text { delay }- \text { SSRT }}{\text { SDRT }},
$$

where ZRFT is the relative finishing times of the stopping and primary-task processes, expressed as a $Z$ score; MRT is the mean primary-task response time; delay is the interval between the stop-signal stimulus and the primary-task response; SSRT is the estimated stop-signal response time; and SDRT is the standard deviation of the primary-task response times (see Logan \& Cowan, 1984; Logan et al., 1984). ${ }^{2}$ If the inhibition functions from different age or diagnostic groups cannot be aligned by plotting them as a function of ZRFT, then we conclude that the shallower functions represent deficiencies in the executive process of inhibition; either the inhibitory process has more variability, or it is triggered less often in the groups with the shallower slope.

\section{Estimates of Stop-Signal Reaction Time}

The race model suggests a way to estimate the time needed to respond to the stop signal at any given delay. Although the SSRT is not observable directly, it may be defined with respect to the distribution of primary-task reaction time (RT). SSRT can be treated as a constant that appears as a point on the primary-task RT distribution. Reactions occurring before this point will not be inhibited, but those coming after it will be inhibited. The proportion of the distribution preceding this point represents the probability of responding despite a stop signal; the proportion following it represents the probability of inhibition. The point may be estimated from the percentile value of the primary-task RT distribution that corresponds to one minus the probability of inhibition. This RT represents the completion of the stopping process relative to the primary-task stimulus. To express the completion of the stopping process relative to the stop-signal stimulus (i.e, to calculate SSRT), the stop-signal delay must be subtracted (for a formal derivation, see Logan \& Cowan, 1984). Younger and disturbed children would be expected to show longer SSRTs than older and normal children. Longer SSRTs in disturbed children compared with those in normal children of similar age and IQ can be viewed as evidence of a deficiency in inhibitory control.

\section{Experiment 1: Normal Development}

\section{Method}

Subjects. Thirty-six boys (12 from each of Grades 2, 4, and 6) and 12 adult volunteers (research assistants, students) were subjects in this study. The children were recruited from three schools through a twostage procedure. First, teachers selected children who they believed were of average intelligence and without learning or behavioral problems. Second, the absence of learning or behavior problems was confirmed through behavior ratings completed by the child's teacher and parents and through measures of intelligence and academic attainment. Informed consent for the experiment was obtained from the children's parents, and assent was obtained from each subject. Parents and teachers completed (a) the appropriate version of the Rutter Behavior Rating Scale (Form A for parents and Form B for teachers; Rutter, 1967; Rutter, Tizard, \& Whitmore, 1970), (b) the SNAP questionnaire (Pelham, Atkins, \& Murphy, 1981), and (c) the Abbreviated Conners Teacher Rating Scales (ACTRS; Conners, 1973). To be included in the sample, children had to obtain scores within the normal range on both parent and teacher questionnaires. More specifically, children had to obtain the following scores: (a) less than 9 on the Rutter-B and less than 13 on the Rutter-A rating scales (scores greater than these indicate psychiatric disturbance); (b) less than 4 on the Inattentive subscale, less than 4 on the Impulsive subscale, and less than 3 on the Hyperactive subscale of the SNAP questionnaire (higher scores, obtained by $5 \%$ of 10-year-old boys, indicate a diagnosis of hyperactivity; Pelham et al, 1981); (c) less than 15 on the ACTRS (scores above 15 are indicative of a diagnosis of hyperactivity; Goyette, Conners, \& Ulrich, 1978).

To confirm that each child was of at least average intelligence, we administered two subtests (Vocabulary and Block Design) of the Wechsler Intelligence Scale for Children-Revised (WISC-R) (Wechsler, 1974) that correlate most highly with the full-scale score. The Wide Range Achievement Test (WRAT-R; Jastak \& Wilkinson, 1984) was used to estimate level of academic attainment. To be included in the study, children had to have full-scale IQ scores of 80 or more and score above the 25 th percentile on the WRAT-R Reading, Spelling, and Arithmetic tests. The mean age of the three groups of students was 95,118 , and 145 months, respectively, and their mean IQs did not differ, $F(2,33)=0.6, n s$. Information on the age and IQ of the adult volunteers was not collected. Subjects were not paid for their participation.

Stopping task. The experimenter-paced stopping task, developed by Logan and colleagues (Logan et al., 1984; Logan \& Cowan, 1984), examines the ability to inhibit responses to a choice reaction-time task in which both speed and accuracy are emphasized. The primary-task stimuli were the uppercase letters $X$ and O, presented by an Apple Ile computer connected to a specialized Cognitive Testing Station (CTS; Digitry Company, Inc., Maine, 1984), which allowed direct and precise control of the stimulus presentation, as well as the collection of response times, with millisecond timing. Each letter, presented one at a time in the center of the screen, was $2 \mathrm{~mm}$ wide and $5 \mathrm{~mm}$ high and, when viewed at a distance of $40 \mathrm{~cm}$, subtended $0.29^{\circ} \times 0.72^{\circ}$ of visual angle. The stop signal was a $1-\mathrm{kHz}$ tone (beep) generated and presented by the computer. Half of the stop signals occurred with an $\mathrm{X}$ and half with an $O$. The details of the stop-signal delays and the manner in which they were generated are presented below.

Each trial began with a fixation point illuminated for $500 \mathrm{~ms}$. It was followed by the letter for that trial, displayed for $1 \mathrm{~s}$ and then extinguished. The screen remained blank for an interval of $1.5 \mathrm{~s}$. Thus, each trial included a period of $2.5 \mathrm{~s}$ in which the subject could respond. The

\footnotetext{
${ }^{2}$ It is not possible to correct for SDRT without prior adjustment for SSRT (Logan et al., 1984).
} 
subject responded by pressing one of two keys on a response box connected to the CTS hardware. Mapping of letters onto the keys was counterbalanced across subjects.

The test trials were presented in blocks of 48 trials; the blocks were organized in three groups of three blocks (total of 432 test trials), with a short break between the groups. The two stimuli occurred equally often in each block, and each stop-signal delay occurred equally often with each letter. Stop signals were presented in $25 \%$ of the trials (108 trials), occurring equally of ten at each of six stop-signal delays, so that a total of 18 stop signals occurred at each stop-signal delay. The sequence of primary-task stimuli, stop signals, and stop-signal delays was random. The trials lasted from 35 to 45 min depending on the length of breaks.

Setting stop-signal delay. To account for differences between subjects in primary-task RT and strategy (e.g., a subject may have held back a response in an attempt to increase the probability of inhibiting), stop-signal delay was defined as the interval between the stop-signal stimulus and the subject's expected primary-task RT. Specifically, MRT was measured in each block of trials, and stop-signal stimuli were presented at delays defined relative to MRT, tracking changes in MRT over time. Logan et al. (1984) showed that various methods of setting the delay produced equivalent results, but tracking MRT produced better inhibition functions. Tracking makes it easier to capture the sloping section of the inhibition function between the high and low asymptotes, which is the most informative part theoretically.

Before the 432 test trials, a practice block of 48 trials was presented to estimate the mean choice response time. During this practice block, stop signals were presented but subjects were not told their purpose. They were presented arbitrarily $500,400,300,200,100$, and $0 \mathrm{~ms}$ before the end of the primary-task stimulus. For each subject, the MRT calculated in the first block was used to set the six stop-signal delays for the second block equal to MRT $-500 \mathrm{~ms}, \mathrm{MRT}-400 \mathrm{~ms}$, MRT -300 $\mathrm{ms}, \mathrm{MRT}-200 \mathrm{~ms}, \mathrm{MRT}-100 \mathrm{~ms}$, and MRT $-0 \mathrm{~ms}$. MRTs for trials without stop signals in the second block were used to set the delays for the third block, and MRTs for the third block were used to set the delays for the fourth, and so on.

Procedure. Adults were tested in the same way as children, except that children were tested at school and adults at the hospital. Subjects were tested individually. They were seated comfortably in front of the computer screen in a quiet room and instructed to keep a finger of the left hand on the left key and a finger of the right hand on the right key throughout the experiment. The experimenter remained in the room. Instructions for the choice response-time task were given first. Subjects were told to respond as quickly and accurately as possible. After the block of practice trials, they were told not to respond to the primary-task stimulus whenever the beep occurred. They were also told not to wait for the stop signal because it would not occur very often. It was explained that the stop signals would occur in such a way that sometimes subjects would be able to stop their response and sometimes not.

The following dependent variables were measured: reaction time to the primary task for trials without stop signals, errors (pressing for $\mathrm{X}$ when $O$ was presented or vice versa), probability of response inhibition at each stop-signal delay, probability of nonresponse to primary-task trials without a stop signal, and SSRT.

Statistical analysis. In order to examine the shape of each group's inhibition function, analysis of variance (ANOVA) with repeated measures across delay for each age group was performed on the probability of inhibition. An interaction between group and delay would indicate differences in the shape of inhibition functions. These differences were examined more precisely through a comparison of the slope of the inhibition function for each group. Slopes were calculated by fitting regression lines to the inhibition function of each subject. If group differences were observed in the slope, regression lines were fitted to the inhibition functions plotted as a function of ZRFT to determine the extent to which primary-task variability rather than differences in inhibitory control accounted for observed differences in the slope of the inhibition function. The slopes of the regression lines for each subject were entered into a univariate ANOVA.

For other variables, such as mean nonresponses, age groups were compared by univariate ANOVAs. For all measures in which the overall $F$ value was significant, post hoc Newman-Keuls tests (Winer, 1971) were conducted to locate differences between age groups. Finally, the association of age and IQ with these indices of performance across all subjects was assessed through correlation analysis.

\section{Results}

Primary-task processes. Age groups differed in performance on the primary task (Table 1). Older subjects responded more quickly and with less variability; differences were particularly marked between the adult and Grade 6 groups and the younger groups. The groups did not differ in mean percentages of errors or of nonresponses.

Stopping processes. Table 2 presents the mean probability of inhibiting a response to the primary task at each stop-signal delay for each age group. Older subjects inhibited a greater proportion of responses over all delays, but the differences were not significant, $F(3,44)=1.9$.

As predicted by the model, the probability of inhibition increased significantly as stop-signal delay increased, $F(5,220)=$ 126.2, $p<.001$ (Table 2 and Figure 1). All age groups were affected similarly (the interaction between age group and delay for the probability of inhibition was nonsignificant, $F(15$, $220)=1.29$. Moreover, the slope of inhibition functions, $F(3$, $44)=1.00, n s$, did not vary among age groups (Table 1 and Figure 1). Although the adult and Grade 6 groups had shorter mean SSRTs than the younger age groups, the differences were not significant, $F(3,44)=2.4$.

Inhibition functions plotted against MRT minus stop-signal delay differ to some extent in height and slope (Figure 1, left panel). The difference in height may be explained by differences in SSRT, whereas the difference in slope may be due to differences in the variability of SSRT and of primary-task RT. The inhibition functions are even closer when plotted against ZRFT (Figure 1, right panel), which corrects for differences due to SSRT and due to primary-task variability. This finding suggests that some of the differences between age groups may have been due to the slight differences in SSRT.

Across all children, age correlated moderately with SSRT $(r=$ $-.32, p<.05$ ) but not with the probability of inhibition or with slope. IQ also correlated moderately with slope $(r=.29, p<.05)$ but not with the percentage of inhibition or with SSRT.

\section{Discussion}

Developmental changes were evident in the performance in the stop-signal paradigm. Older children and adults responded more quickly to the primary task than did younger children, and they did so with less variability of RT and similar percentages of error.

Development does not significantly affect the ability to inhibit a motor response, as shown by the effect of stop-signal delay, the slope of the inhibition function, and the overall proba- 
Table 1

Performance on the Stop-Signal Paradigm in Experiment 1

\begin{tabular}{|c|c|c|c|c|c|c|c|c|c|c|c|}
\hline \multirow[b]{2}{*}{ Measure } & \multicolumn{2}{|c|}{ Grade 2} & \multicolumn{2}{|c|}{ Grade 4} & \multicolumn{2}{|c|}{ Grade 6} & \multicolumn{2}{|c|}{ Adults } & \multirow[b]{2}{*}{$F(3,44)$} & \multirow[b]{2}{*}{ D } & \multirow{2}{*}{$\begin{array}{c}\text { Newman-Keuls } \\
\text { post hoc test }\end{array}$} \\
\hline & $M$ & $S D$ & $M$ & $S D$ & $M$ & $S D$ & $M$ & $S D$ & & & \\
\hline MRT for correct responses & 963 & 100 & 884 & 135 & 763 & 178 & 660 & 59 & 13.5 & $<.01$ & $2>4>6>$ adult \\
\hline Mean $S D$ of MRT & 240 & 40 & 201 & 40 & 154 & 49 & 109 & 31 & 23.6 & $<.01$ & $2>4>6>$ adult \\
\hline$\%$ of errors & 8.4 & 7.2 & 7.8 & 4.4 & 7.0 & 7.8 & 3.3 & 2.1 & 1.9 & $n s$ & \\
\hline$\%$ of nonresponses & 2.2 & 1.9 & 3.2 & 6.1 & 1.4 & 3.4 & 2.0 & 4.3 & .4 & $n s$ & \\
\hline Slope & 9.9 & 3.4 & 9.2 & 4.1 & 10.3 & 3.7 & 12.0 & 5.1 & 1.0 & $n s$ & \\
\hline Mean SSRT & 326 & 93 & 276 & 81 & 253 & 49 & 264 & 54 & 2.4 & $n s$ & \\
\hline
\end{tabular}

Note, $\mathrm{MRT}=$ mean response time; $\mathrm{SSRT}=$ stop-signal response time.

bility of inhibition. Younger children triggered the stopping process as frequently as older children and adults, and their stopping processes did not differ significantly in speed. The mechanism of inhibitory control thus seems well developed by Grade 2, although it may be less so in younger children.

The low rate of nonresponses to the primary task at all ages excludes the possibility that failure to inhibit simply reflected distractibility or strategy (e.g, failing to attend to the target stimuli or trying to improve the chance of inhibiting by deciding before the trial not to respond regardless of the stimulus).

These findings bring into question the apparent development in inhibitory control and the decrease in impulsivity with increasing age from the preschool years through childhood, changes detected with other tasks, for example, the MFFT (Kogan, 1983). Such changes might result from improvement in executive functions other than inhibitory control, increasing awareness of strategies, or improvement in attentional capacity rather than from development in inhibitory control (see Case \& Globerson, 1974; Kahneman, 1973).

\section{Experiment 2: Childhood Psychopathology}

The second experiment compared performance on the stopping paradigm of groups of children with ADDH, conduct disorder (CD), learning disorder (LD), and emotional disorder (ED) with that of normal children. It also included a group of children with both ADDH and CD because this presentation of childhood hyperactivity is the most common (Stewart, DeBlois, \& Cummings, 1980) and there is evidence that it might have distinct correlates (Schachar \& Wachsmuth, 1990). Perva-

Table 2

Percentage Inhibition by Stop-Signal Delay in Experiment I

\begin{tabular}{ccccc}
\hline $\begin{array}{c}\text { Stop-signal delay } \\
\text { (ms) }\end{array}$ & $\begin{array}{c}\text { Grade } \\
2\end{array}$ & $\begin{array}{c}\text { Grade } \\
\mathbf{4}\end{array}$ & $\begin{array}{c}\text { Grade } \\
6\end{array}$ & Adults \\
\hline MRT - 500 & 61 & 60 & 66 & 73 \\
MRT - 400 & 48 & 59 & 61 & 76 \\
MRT - 300 & 47 & 46 & 48 & 64 \\
MRT - 200 & 32 & 29 & 34 & 41 \\
MRT - 100 & 18 & 28 & 26 & 30 \\
MRT - 0 & 12 & 18 & 18 & 22 \\
\hline
\end{tabular}

Note. MRT $=$ mean response time. sively and situationally hyperactive ADDH subjects were also distinguished because evidence suggests that their cognitive function may be distinct (Chee, Logan, Schachar, Lindsay, \& Wachsmuth, 1989; Sandberg, Rutter, and Taylor, 1978). The ADDH group was expected to show deficient inhibitory control compared with the normal control (NC) group (i.e, to have a lower or flatter inhibition function). If an impulse control deficit was specific to the ADDH group, this group's inhibition function would also be lower or flatter than that of the pathological control groups.

\section{Method}

Subjects. The subjects in this experiment were 60 children referred for psychiatric or psychoeducational assessment of learning or behavioral disorders to the outpatient Departments of Psychiatry or Psychology, The Hospital for Sick Children, Toronto, Canada, and 10 normal volunteers recruited through an advertisement in a local home and school association newspaper. Subjects were compensated for their travel expenses. Children were excluded from the study if they showed evidence of a neurological disorder such as epilepsy, had a history or evidence of psychosis, or had an IQ of less than 80 . All children had to be free of any medication for a minimum of 48 hours preceding testing.

Assessment of the child. The subjects were assigned a diagnosis on the basis of a semistructured diagnostic interview with the parent(s), behavior ratings completed by each child's classroom teacher, individual assessment, and psychoeducational assessment.

Information was obtained by one of two child psychiatrists from each child's parent(s) by following an interview protocol designed to elicit symptoms relevant to diagnoses from the third edition of the Diagnostic and Statistical Manual of Mental Disorders (DSM-III; American Psychiatric Association, 1980). The interview covered prenatal, birth, postnatal, developmental, medical, academic, and family histories, as well as current child behavior and symptoms of psychopathology. It also investigated the quality of the child's current interaction with peers, siblings, and adults. For each setting (e.g., at play out of doors, in stores, with other children), parents were asked to describe a recent example of their child's behavior. Parents' subjective statements about behavior were not considered; instead, each symptom was rated by the interviewer on the basis of its severity, age appropriateness, and resultant degree of handicap. Only behaviors that were severe, handicapping, and age-inappropriate were considered diagnostic symptoms. This interview was developed because we found that assessment based on a detailed description of child behavior rather than on questionnaires or structured interviews was necessary to distinguish symptoms of hyperactivity from those of conduct disorder. Interrater reli- 
ability of this interview was assessed by having a second child psychiatrist complete ratings of 18 audiotaped interviews. Raters agreed on the presence or absence of $97 \%$ of symptoms $(x=.92)$. In no case did disagreement about individual symptoms result in disagreement about the diagnosis.

The classroom teacher of each child completed the Rutter-B Rating Scale (Rutter, 1967), the ACTRS (Conners, 1973), and the SNAP questionnaire (Pelham et al, 1981). Each child was interviewed by a psychologist following the format of Rutter and Graham (1968). In the absence of accepted criteria for combining parent and child information for research diagnoses, children were excluded from the study if the results of the child interview were markedly divergent from those of the parent interview. This occurred in the case of one child with pervasive developmental disorder.

Psychoeducational assessment. The psychoeducational assessment consisted of four subtests from the WISC-R (Block Design, Object Assembly, Similarities, and Vocabulary; Wechsler, 1974) and of the WRAT-R (Jastak \& Wilkinson, 1984). If these tests had been administered to the child within the previous year, they were not given again.

Child diagnosis. Diagnoses were assigned according to DSM-III criteria, and multiple diagnoses were permitted. Because a diagnosis of ADDH does not require agreement between parents and teacher, ADDH could be diagnosed if either the parental interview or the teacher report indicated its presence. An interview-based diagnosis of ADDH was made if the parents reported three symptoms of inattentiveness, three of impulsivity, and two of hyperactivity in addition to a history of hyperactivity, impulsivity, and inattentiveness before the age of 6 years.

A teacher-based diagnosis of ADDH was made if the teacher rated the child as disturbed and significantly hyperactive. A rating of 9 or more on the Rutter-B Rating Scale was taken as an indication of psychiatric disturbance (Rutter et al, 1970), whereas the presence of any two of the following three criteria was considered evidence of clinically significant hy peractivity: (a) a rating of at least 5 out of 6 on the RutterB Hyperactivity factor, a score obtained by $3 \%$ of 10 -year-old boys (Schachar, Rutter, \& Smith, 1981); (b) a rating of 4 Inattentive, 4 Impulsive, and 3 Hyperactive items on the SNAP questionnaire, a score obtained by $5 \%$ of 10-year-old boys (Pelham et al., 1981); and (c) an abbreviated score of 15 or more on the ACTRS, a score predictive of a clinical diagnosis of hyperactivity (Goyette et al., 1978). Children who received both an interview- and a teacher-based diagnosis were considered pervasively hyperactive. However, from previous experience (Chee et al., 1989), we expected to find that only 50\% of the ADDH group was pervasively hyperactive. The few cases of attention deficit disorder without hyperactivity were excluded from the study.

Children were assigned to the CD group if they met the DSM-III criteria for it, based on the parent-interview protocol. In addition, the diagnosis of $C D$ was assigned to children diagnosed with oppositional disorder (OD) whose symptoms were severe and pervasive, involving relationships with both parents or a variety of adults. Of the 42 children in the $C D$ and $A D D H+C D$ groups, 22 were diagnosed with $C D$ and 20 with $\mathrm{OD}$. Of the $22 \mathrm{CD}$ diagnoses, 14 were aggressive and 8 nonaggressive. Typically, the aggressive children presented with longstanding symptoms of repetitive physical aggression toward peers and of vandalism; the nonaggressive children showed a pattern of habitual lying and stealing. The majority of the OD group had more than the minimum number of symptoms (two) for the diagnosis and had oppositional symptoms that affected relationships with family and other adults, as well as with peers and siblings. The practice of combining $C D$ and OD groups is consistent with the observation that the two diagnoses have not been differentiated and are qualitatively similar (Anderson, Williams, McGee, \& Silva, 1987; Reeves, Werry, Elkind, \& Zametkin, 1987; Werry, Methven, Fitzpatrick, \& Dixon, 1983).

A diagnosis of ED was assigned to children meeting the DSM-III criteria for any of the following: separation anxiety or overanxious,
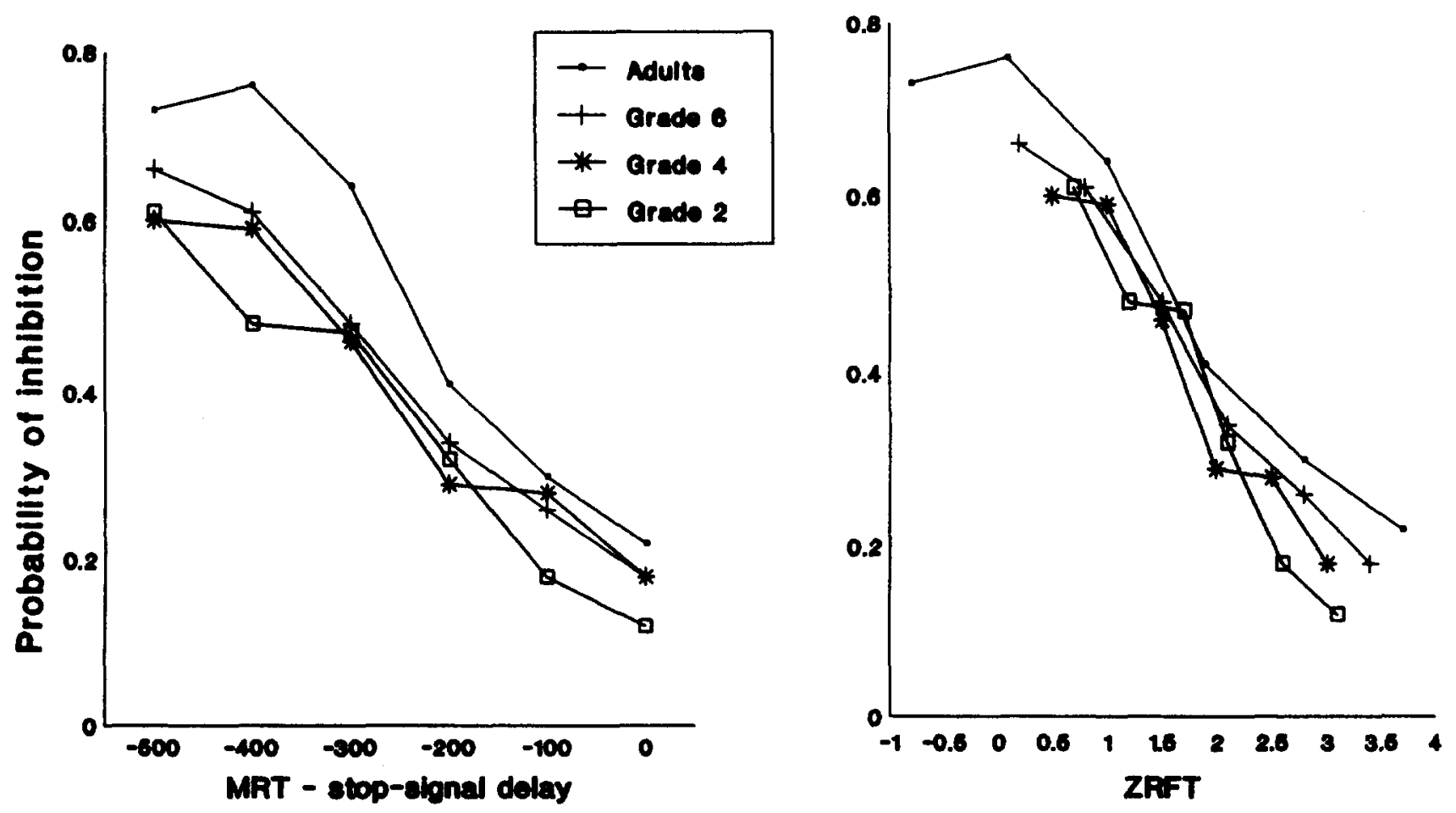

Figure 1. Probability of inhibition of age groups by MRT minus stop-signal delay (left panel) and ZRFT (right panel). (MRT = mean response time; $Z R F T=Z$ score, relative finishing time.) 
phobic, obsessive compulsive, or somatization disorders. LD was diagnosed if the full-scale IQ was average or higher and the standard score on the WRAT Reading subtest was below the 25th percentile in the absence of a physical or sensory disorder (Siegel \& Heaven, 1986).

On the basis of these criteria, the children were assigned to one of five diagnostic groups: ADDH, CD, mixed ADDH + CD, ED, or $L D$. Multiple diagnoses were permitted. Children in the $C D, E D$, and $L D$ groups could not be hyperactive according to the criteria used, but those in the CD and ED groups could have an additional diagnosis of LD. Children with ADDH, ADDH + CD, or CD could have an additional diagnosis of ED or LD. Volunteers qualified as controls (NC) only if they were free of any diagnosis.

The child diagnostic interview administered to parents did not include two of the new criteria for oppositional defiant disorder contained in the revised edition of the DSM-III (DSM-III-R; American Psychiatric Association, 1987) but did include all items necessary for revised diagnoses of $\mathrm{ADDH}, \mathrm{CD}$, and $\mathrm{ED}$. All subjects in the latter three groups met criteria for the equivalent DSM-III-R diagnosis. Although not every item for oppositional defiant disorder was included, it was our clinical impression that all OD subjects would merit a $D S M$ III-R diagnosis of oppositional defiant disorder.

Stopping task. The task used in Experiment 2 differed from that used in Experiment 1 only by the addition of a block of practice trials. This additional practice block consisted of practice on the primary task, as did the practice block in Experiment 1, but no stop signals were presented. In Experiment 2, the second practice block presented subjects with both the primary task and stop signals to provide practice in stopping. These two practice blocks were followed by nine experimental blocks, as in Experiment 1. The MRT for each block was based on the MRT to the primary task of the preceding block. Stimuli were presented on the same computer and in the same way for both experiments, and stop signals were randomized across conditions in the same way. The experimental procedure was identical except that all testing was conducted at the hospital while the child's parents were being interviewed.

Statistical analysis. The analyses were conducted in the same way as for Experiment 1. Because differences in inhibition were not expected between all of the groups, planned comparisons were conducted to compare the ADDH group with the NC group and each of the other groups (Kirk, 1982 pp. 832-839).

\section{Results}

The diagnostic groups did not differ significantly in mean age (Table 3) or in mean IQ, although the NC group had somewhat higher mean IQs than the other groups.

Primary task processes. The diagnostic groups did not differ in MRT to the primary task (see Table 3). However, groups differed significantly in the variability of MRT. The ADDH group showed the greatest amount of variability in MRT and the ED group the least, but the intergroup differences tested post hoc did not reach conventional levels of significance. The ADDH group made more errors on the primary task, but the difference was not significant. The rate of nonresponse did not differ among diagnostic groups.

Stopping processes. Although the mean probability of inhibiting a response after a stop signal varied from $48 \%$ in the $\mathrm{NC}$ group to $28 \%$ in the ADDH group (Table 4), it did not differ significantly among the groups, as is evident in the absence of a main effect for group in an ANOVA with factors for diagnosis and stop-signal delay, $F(5,64)=1.5$, ns. However, planned comparison indicated the expected intergroup differ- ences in the probability of inhibition: The ADDH group inhibited fewer responses than the NC group, $F(1,64)=6.97, p<.05$, but not fewer than the CD or ADDH + CD groups or all pathological groups taken together.

As predicted, the probability of inhibiting a response was strongly affected by the stop-signal delay, $F(5,329)=121.2, p<$ .001 . The probability fell from about $60 \%$ at the longest delays to about $15 \%$ at the shortest (Table 4 and Figure 2, left panel). Variation in the stop-signal delay affected the probability of inhibition of the diagnostic groups differently, as is evident in a significant interaction between group and delay, $F(25,320)=$ $2.4, p<.01$. Post hoc comparisons revealed that the effect of delay was significantly less in the ADDH group than in any other group: $F(5,320)=9.7, p<.001$, for the NC group; $F(5$, $320)=5.3, p<.001$, for the pathological groups considered together; $F(5,320)=5.0, p<.001$, for the ADDH + CD group; and $F(5,320)=2.9, p<.05$, for the $\mathrm{CD}$ group.

The smaller effect of delay on the probability of inhibition in the ADDH group was also evident in the analysis of the slopes of the regression lines fitted to the probability of the inhibition data (Table 3 and Figure 2), indicating that the ADDH group was affected by delay less than the other groups, $F(5,64)=3.0$, $p<.05$. Post hoc comparisons indicated that the slope of the ADDH group's results was less than that of the NC group $(p<.05)$.

The probability of inhibition is plotted against ZRFT in the right panel of Figure 2, correcting for differences in primarytask variability and SSRT. In this case, the ZRFT correction appears to enhance the differences between the groups' inhibition functions. The ADDH group inhibited much less often than did clinical controls at equivalent values of ZRFT. The differences in slope between ADDH subjects and normal controls seem less pronounced than when they are plotted against MRT minus the stop-signal delay, but they remain apparent.

These conclusions were confirmed by ANOVA. Group differences in the slope of the inhibition functions plotted against ZRFT did not reach conventional levels of significance, $F(5$, $64)=1.49$, although the slope of the ADDH group remained much flatter than that of the other groups. This observation was confirmed by the planned comparisons indicating that the ADDH group had a flatter slope than either the NC, $F(1,64)=$ $5.2, p<.05$, or the ADDH $+\mathrm{CD}, F(1,64)=4.9, p<.05$, groups. These slope differences indicate that greater variation in the primary-task RT cannot completely explain the observed deficit in inhibitory control of the ADDH group.

Deficient inhibitory control was also evident in the differences in SSRT among the groups. The mean SSRT of the NC group was the shortest and that of the ADDH group the longest. The main effect of group did not reach conventional levels of significance, $F(5,62)=2.3, p=.06$, but planned comparisons indicated that mean SSRT was longer for the ADDH group than for the NC group, $F(1,62)=9.1, p<.01$. However, these differences in SSRT were insufficient to account for group differences in inhibition functions because these functions were not aligned when plotted against ZRFT (i.e, there were still large intercept differences).

Across all subjects, age correlated weakly with the percentage of inhibition $(r=.20)$ and the slope $(r=.22)$ and moderately with the mean SSRT $(r=-.29, p<.05)$. IQ was also weakly asso- 
Table 3

Performance on the Primary Task of Subjects in Each Diagnostic Group in Experiment 2

\begin{tabular}{|c|c|c|c|c|c|c|c|c|c|c|c|c|c|c|}
\hline \multirow[b]{2}{*}{ Measure } & \multicolumn{2}{|c|}{$\mathrm{NC}$} & \multicolumn{2}{|c|}{ LD } & \multicolumn{2}{|c|}{ ED } & \multicolumn{2}{|c|}{$\mathrm{CD}$} & \multicolumn{2}{|c|}{$\underset{\mathrm{CD}}{\mathrm{ADDH}}+$} & \multicolumn{2}{|c|}{ ADDH } & \multirow[b]{2}{*}{$F(5,64)$} & \multirow[b]{2}{*}{$p$} \\
\hline & $M$ & $S D$ & $M$ & $S D$ & $M$ & $S D$ & $M$ & $S D$ & $M$ & $S D$ & $M$ & $S D$ & & \\
\hline Age (months) & 120 & .14 & 120 & 14 & 119 & 16 & 117 & 23 & 112 & 17 & 111 & 21 & .6 & $n s$ \\
\hline IQ & 123 & 16 & 112 & 18 & 107 & 12 & 110 & 17 & 104 & 12 & 107 & 14 & 2.3 & $n s$ \\
\hline MRT for correct responses & 901 & 131 & 889 & 117 & 845 & 146 & 920 & 146 & 952 & 163 & 901 & 213 & .7 & $n s$ \\
\hline Mean $S D$ of MRT & 194 & 62 & 196 & 53 & 188 & 51 & 231 & 35 & 235 & 74 & 255 & 78 & 2.4 & $<.05$ \\
\hline Slope ${ }^{\mathrm{a}}$ & 13.7 & 6.8 & 10.1 & 6.3 & 9.3 & 6.0 & 9.4 & 3.9 & 10.4 & 4.9 & 5.2 & 3.7 & 3.0 & $<.05$ \\
\hline ZRFT slope & 23.9 & 12.6 & 18.5 & 10.2 & 17.8 & 12.9 & 21.5 & 10.8 & 22.7 & 10.0 & 12.9 & 12.4 & 1.5 & $n s$ \\
\hline Mean SSRT & 269 & 142 & 309 & 94 & 297 & 98 & 322 & 75 & 328 & 145 & 437 & 183 & 2.3 & $n s$ \\
\hline
\end{tabular}

Note. $\mathrm{NC}=$ normal controls $(n=10) ; \mathrm{LD}=$ learning disorder $(n=11) ; \mathrm{ED}=$ emotional disorder $(n=13) ; \mathrm{CD}=\operatorname{conduct}$ disorder $(n=9) ; \mathrm{ADDH}=$ attention deficit disorder with hyperactivity $(n=13$; for $\mathrm{ADDH}+\mathrm{CD}, n=14)$; MRT = mean response time; ZRFT $=Z$ score, relative finishing time; SSRT = stop-signal response time.

A Newman-Keuls post hoc test for slope showed ADDH $<$ NC.

ciated with the percentage of inhibition (17) and the mean SSRT $(-.03)$, but it correlated moderately with the slope $(r=.34$, $p<.01)$.

$I Q$ and inhibitory control. Even though the diagnostic groups did not differ in IQ, the possible confounding effect of IQ on group differences in inhibition was investigated because of the significant correlation between IQ and slope. The interaction between the covariate IQ and group was not significant, $F(5,58)=1.7$, indicating that the relationship of IQ and slope is similar across groups (Kerlinger \& Pedhazur, 1973). In an analysis of covariance with $\mathrm{lQ}$ as covariate, the intergroup differences in slope were significant, $F(5,63)=75.6, p<.05$, whereas the effect of IQ was not, $F(1,63)=2.17$. Covariation for IQ reduced the difference between ADDH- and NC-adjusted group means, but the difference remained significant (Kerlinger \& Pedhazur, 1973). Adjustment for IQ did not greatly alter the slopes of the results of the pathological controls; these groups had IQ-adjusted slopes that did not differ significantly from those of the NC or ADDH groups.

Situational versus pervasive hyperactivity. As expected, only 5 of 13 children meeting the criteria for a diagnosis of ADDH

Table 4

Percentage Inhibition by Stop-Signal Delay in Experiment 2

\begin{tabular}{ccccccc}
\hline & \multicolumn{6}{c}{ Diagnostic group } \\
\cline { 2 - 7 } $\begin{array}{c}\text { Stop-signal delay } \\
\text { (ms) }\end{array}$ & NC & LD & ED & CD & ADDH + CD & ADDH \\
\hline MRT - 500 & 76 & 58 & 59 & 60 & 61 & 43 \\
MRT - 400 & 70 & 53 & 53 & 58 & 61 & 36 \\
MRT - 300 & 62 & 44 & 44 & 34 & 50 & 29 \\
MRT - 200 & 43 & 27 & 30 & 34 & 34 & 21 \\
MRT - 100 & 22 & 20 & 23 & 22 & 22 & 25 \\
MRT - 0 & 13 & 11 & 16 & 16 & 14 & 16 \\
\hline
\end{tabular}

Note $. \mathrm{NC}=$ normal controls; $\mathrm{LD}=$ learning disorder; $\mathrm{ED}=$ emotional disorder; $\mathrm{CD}=$ conduct disorder; $\mathrm{ADDH}=$ attention deficit disorder with hyperactivity; $\mathrm{MRT}=$ mean response time. met the criteria both at home and at school. The performance on the stopping task of these two subgroups was quite different. Pervasively hyperactive children inhibited on only $13 \%$ of stopsignal trials averaged across all delays, in contrast to $38 \%$ for the situationally hyperactive group, $F(1,11)=9.1, p<.05$, and had a significantly flatter inhibition function with a slope of 2.4 , compared to that of 6.9 for the situationally hyperactive subgroup, $F(1,11)=6.0, p<.05$. When the 5 pervasively hyperactive subjects were excluded from group comparison, intergroup differences in slope were no longer significant, $F(5,59)=1.41$.

\section{Discussion}

Our findings indicate that these diagnostic groups do not differ in the executive processes that determine performance on the primary task. And, with the exception of the ADDH group, all subjects controlled their actions closely and inhibited them on command with great speed. Differences in inhibition functions remained after the ZRFT correction, indicating that the inhibition mechanism of the ADDH group was triggered less frequently or was substantially more variable, as well as slower. Moreover, intergroup variation in IQ did not account for differences in inhibitory control.

These results support the validity of the narrowly defined syndrome of pervasive hyperactivity rather than that of the more broadly defined ADDH diagnosis (see also Chee et al, 1989; Sandberg et al, 1978; Schachar et al., 1981; Taylor et al, 1986). At the same time, deficient inhibitory control cannot simply be attributed to the severity of disturbance: The mixed ADDH + CD group had the greatest number of symptoms of psychopathology yet did not have the most severe deficit in inhibitory control.

This is the second experiment in which we have observed that the ADDH + CD group does not demonstrate the same cognitive deficit as the ADDH group (Chee et al, 1989). Differences between these two subgroups have also appeared in studies of their clinical features. The mixed group is associated with a greater incidence of psychosocial adversity and parental psy- 

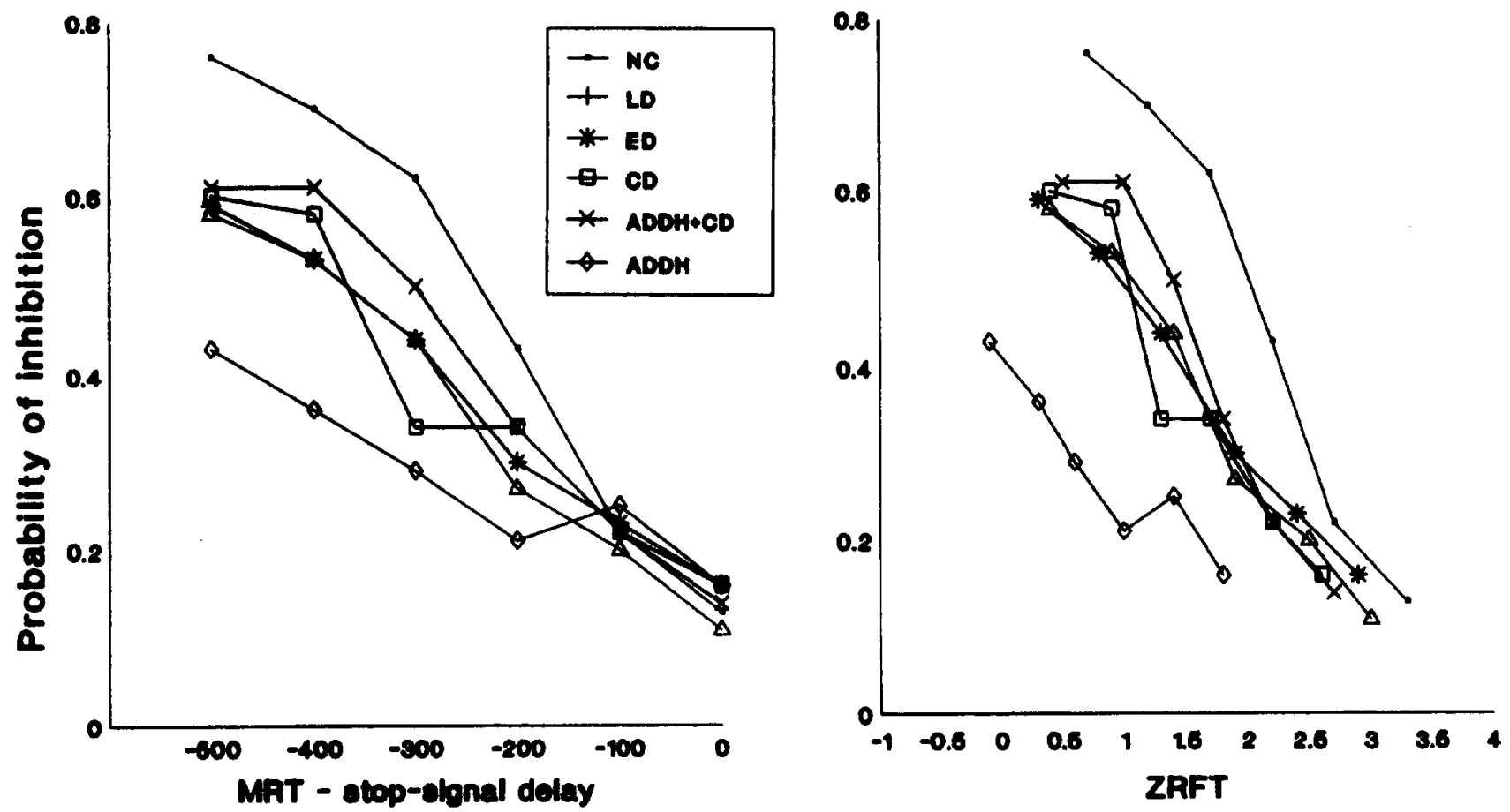

Figure 2. Probability of inhibition of diagnostic groups by MRT minus stop-signal delay (left panel) and ZRFT (right panel). $(\mathrm{NC}=$ normal controls; $\mathrm{LD}=$ learning disability; $\mathrm{ED}=$ emotional disorder; $\mathrm{CD}=$ conduct disorder; $\mathrm{ADDH}=$ attention deficit disorder with hyperactivity; $\mathrm{MRT}=$ mean response time; ZRFT $=Z$ score, relative finishing time.)

chopathology (Biederman, Munir, \& Knee, 1987; Schachar \& Wachsmuth, 1990; Szatmari, Offord, \& Boyle, 1989) and a worse prognosis (Schachar et al., 1981). Taken together, these studies support the contention that the hyperactivity of children with a diagnosis of $\mathrm{CD}$ may be qualitatively different from the hyperactivity of children without it. The former might represent a correlate of psychosocial disturbance, whereas the latter might reflect a cognitive disturbance.

\section{General Discussion}

The results of these experiments confirm the predictions of the model of inhibitory control on which the stop-signal paradigm is based (Logan \& Cowan, 1984) and reflect the utility of the paradigm as a measure of inhibitory control. The paradigm explains impulsivity in terms of underlying processes rather than describing only surface features. It allows researchers to measure inhibitory control directly by analyzing inhibition functions and estimating stop-signal reaction time.

As the model predicted, variation in the delay between the occurrence of the stop signal and the subject's MRT affected the probability of inhibition in both studies. With longer delays, the probability increased. The finding of Experiment 1 that development in the stopping and primary-task processes can occur independently also confirms the model. This model assumes that the two processes (stopping and response) are independent and do not compete for resources. Moreover, the dynamic tracking, which is part of the stopping paradigm, allows for the assessment of inhibitory control independently of
MRT. MRT may reflect strategy or attentional capacity rather than inhibitory control.

The analysis of the performance of normal and disturbed subjects in the stop-signal paradigm helps us to understand the development of the executive function of inhibitory control in normal children and confirms the central role of deficient impulse control in the pervasively hyperactive subgroup of ADDH subjects. Contrary to what previous research might have predicted, the inhibitory control of younger children did not differ significantly from that of older children. The observed improvement with increasing age in the primary-task process in Experiment 1 suggests that the executive functions involved in the selection, execution, or maintenance of an optimal response strategy develop with age. Improvement in these functions rather than in inhibitory control may have led to the improvement in performance on tasks such as the MFFT.

The stopping task differs from tasks requiring impulse control that a child might confront in everyday life. The stop signal is salient and easily detected. Real-world stop signals may be harder to discriminate. Consequently, inhibitory control could develop as a consequence of development in discrimination. Nevertheless, these results indicate that the ability to inhibit action after the signal is detected is well developed by Grade 2 and does not develop much beyond that.

The stop-signal paradigm also shows that a specific deficit in inhibitory control might underlie the impulsivity of pervasively hyperactive children. This deficit may be evident in a range of behaviors, such as failure to stop, look, and listen; failure to inhibit attention to distracting stimuli; and proneness to acci- 
dents. The central role of deficient inhibitory control was also evident in another study: We found that improvements in behavior and academic performance resulting from treatment with methylphenidate were strongly associated with improvement in inhibitory control as measured by the stop-signal paradigm (Tannock, Schachar, Carr, Chajczyk, \& Logan, 1989). Compared with a placebo, treatment with methylphenidate was associated with the increased probability of inhibiting a response, increased slope of inhibition functions, and decreased SSRT.

The stopping paradigm contrasts with the MFFT and other measures of impulsivity in which performance depends on factors such as MRT and strategy, as well as on inhibitory control. In addition, the probability of inhibition on the stopping task is not as strongly correlated with $\mathrm{IQ}$ as is the performance on other popular measures of impulsivity such as the MFFT, Porteus mazes, or draw-a-line-slowly tasks (Milich \& Kramer, 1984). Furthermore, in the study of Tannock et al. (1989), the effects of methylphenidate on MFFT performance were less clear than its effects on stop-signal paradigm performance, suggesting that MFFT latency and error measures are less sensitive to medication effects than are measures derived from the stopsignal paradigm.

Whereas these observations bear on the inhibition of discrete motor actions, research with adults indicates remarkable consistency in the degree of control across a range of motor, speech, and cognitive tasks (Logan \& Cowan, 1984; Logan, 1985). Further research will be necessary to determine whether the properties of control are as consistent in children. However, it seems reasonable to assume that these results can be generalized to other tasks and situations.

\section{References}

American Psychiatric Association. (1980). Diagnostic and statistical manual of mental disorders (3rd ed.). Washington, DC: Author.

American Psychiatric Association. (1987). Diagnostic and statistical manual of mental disorders (3rd ed., rev). Washington, DC: Author.

Anderson, J. C., Williams, S., McGee, R., \& Silva, P. A. (1987). DSM-III disorders in preadolescent children: Prevalence in a large sample from the general population. Archives of General Psychiatry, 44, 6976.

Ault, R. L., Crawford, D. E., \& Jeffrey, W. E. (1972). Visual scanning strategies of reflective, impulsive, fast-accurate, and slow-accurate children on the Matching Familiar Figures test. Child Development, 43, 1412-1417.

Biederman, J., Munir, K., \& Knee, D. (1987). Conduct and oppositional disorder in clinically referred children with attention deficit disorder: A controlled family study. Journal of the American Academy of Child and Adolescent Psychiatry, 26, 724-727.

Brown, A. L., Bransford, J. D, Ferrara, R. A., \& Campione, J. C. (1983). Learning, remembering, and understanding. In P. H. Mussen (Ed), Handbook of child psychology (Vol. 3, pp. 77-166). New York: Wiley.

Campbell, S. B., Douglas, V. I., \& Morgenstern, G. (1971). Cognitive styles in hyperactive children and the effect of methylphenidate. Journal of Child Psychology and Psychiatry, 12, 55-67.

Case, R., \& Globerson, T. (1974). Field independence and central computing space. Child Development, 45, 772-778.

Chee, P, Logan, G., Schachar, R., Lindsay, P., \& Wachsmuth, R. (1989). Effects of event rate and display time on sustained attention in hyperactive, normal, and control children. Journal of Abnormal Child Psychology, 17, 371-391.
Conners, C. K. (1973). Rating scales in drug studies with children. Psychopharmacy Bulletin [Special issue: Pharmacotherapy with children], 24-29.

Douglas, V. I. (1983). Attentional and cognitive problems. In M. Rutter (Ed.), Developmental neuropsychiatry (pp. 280-329). New York: Guilford Press.

Douglas, V. I., \& Peters, K. G. (1979). Toward a clearer definition of the attentional deficit of hyperactive children. In G. A. Hale and $\mathbf{M}$. Lewis (Eds.), Attention and cognitive development (pp. 173-248). New York: Plenum Press.

Goyette, C. H., Conners, C. K., \& Ulrich, R. F. (1978). Normative data on revised Conners Parent and Teacher Rating Scales. Journal of Abnormal Child Psychology, 6, 221-236.

Jastak, S., \& Wilkinson, G. S. (1984). The Wide Range Achievement Test: Administration manual (rev). Wilmington, DE: Jastak Associates.

Kagan, J, Rosman, B. L, Day, D., Albert, J, \& Phillips, W. (1964). Information processing in the child: Significance of analytic and reflective attitudes. Psychological Monographs, 78(1, Whole No. 578).

Kahneman, D. (1973). Attention and effort. Englewood Cliffs, NJ: Prentice-Hall.

Kerlinger, F. N., \& Pedhazur, E. J. (1973). Multiple regression in behavioral research. New York: Holt, Rinehart \& Winston.

Kirk, R. (1982). Experimental design: Procedures for behavioural sciences. Pacific Grove, CA: Brooks/Cole.

Kogan, N. (1983). Stylistic variation in childhood and adolescence: Creativity, metaphor, and cognitive style. In P. Mussen (Ed), Handbook of child psychology: Vol. 2. Cognitive development (pp. 630-706). New York: Wiley.

Logan, G. D. (1985). Executive control of thought and action. Acta Psychologia, 60, 193-210.

Logan, G. D., \& Cowan, W. B. (1984). On the ability to inhibit thought and action: A theory of an act of control. Psychological Review, 91, 295-327.

Logan, G. D., Cowan, W. B., \& Davis, K. A. (1984). On the ability to inhibit simple and choice reaction time responses: $A$ model and a method. Journal of Experimental Psychology: Human Perception and Performance, 10, 276-291.

Luce, R. D. (1986). Response times. New York: Oxford University Press.

Milich, R., \& Kramer, J. (1984). Reflections on impulsivity: An empirical investigation of impulsivity as a construct. Advances in Learning and Behavioural Disabilities, 3, 57-94.

Pelham, W. E., Atkins, M., \& Murphy, H. A. (1981, September). ADD with and without hyperactivity: Parent, teacher, and peer rating correlates. In W. E. Pelham (Chair), DSM-III category of attention deficit disorder: Rationale, operation, and correlates. Symposium conducted at the 89th Annual Convention of the American Psychological Association, Los Angeles.

Peloquin, L. J., \& Klorman, R. (1986). Effects of methylphenidate on normal children's mood, event-related potentials, and performance in memory scanning and vigilance. Journal of Abnormal Psychology, 95, 88-98.

Reeves, J. C., Werry, J. S., Elkind, G. S., \& Zametkin, A. (1987). Attention deficit, conduct oppositional, and anxiety disorders in children: II. Clinical characteristics. Journal of the American Academy of Child and Adolescent Psychiatry, 26, 144-155.

Rutter, M. (1967). A children's behaviour questionnaire for completion by teachers: Preliminary findings. Journal of Child Psychology and Psychiatry, 8, 1-11.

Rutter, M., \& Graham, P. (1968). The reliability and validity of the psychiatric assessment of the child: I. Interview with the child. British Journal of Psychiatry, 114, 563-579.

Rutter, M., Tizard, J., \& Whitmore, K. (1970). Education, health and 
behaviour: Psychological and medical study of child development. New York: Wiley.

Salkind, N. J., \& Wright, J. C. (1977). The development of reflectionimpulsivity and cognitive efficiency. Human Development, 20,377387.

Sandberg, S. T, Rutter, M., \& Taylor, E. (1978). Hyperkinetic disorder in psychiatric clinic attenders. Developmental Medical Child Neurology, 20, 279-299.

Schachar, R., Rutter, M., \& Smith, A. (1981). The characteristics of situationally and pervasively hyperactive children: Implications for syndrome definition. Journal of Child Psychology and Psychiatry, 22, 375-392.

Schachar, R., \& Wachsmuth, R. (1990). Hyperactivity and parental psychopathology. Journal of Child Psychology and Psychiatry, 31, 381-392.

Siegel, L. S. \& Heaven, R. K. (1986). Categorization of learning disabilities. In S. Ceci (Ed), Handbook of cognitive, social, neuropsychological aspects of learning disabilities (Vol. 1, pp. 95-121). Hillsdale, NJ: Erlbaum.

Stewart, M. A., DeBlois, C. S., \& Cummings, C. (1980). Psychiatric disorder in the parents of hyperactive boys and those with conduct disorder. Journal of Child Psychology and Psychiatry, 21, 283-292.
Szatmari, P., Offord, D., \& Boyle, M. (1989). Ontario Child Health Study: Prevalence of attention deficit disorder with hyperactivity Journal of Child Psychology and Psychiatry, 30, 219-230.

Tannock, R., Schachar, R. J., Carr, R. P, Chajczyk, D, \& Logan, G. D. (1989). Effects of methylphenidate on inhibitory control in hyperactive children. Journal of Abnormal Child Psychology, 17, 473-491.

Taylor, E., Everitt, B., Thorley, G., Schachar, R., Rutter, M., \& Wieselberg, M. (1986). Conduct disorder and hyperactivity: II. A cluster analytic approach to the identification of a behavioural syndrome. British Journal of Psychiatry, 149, 768-777.

Wechsler, D. (1974). Wechsler Intelligence Scale for Children: Manual (rev). New York: Psychological Corporation.

Werry, J. S., Methven, R. J., Fitzpatrick, J, \& Dixon, H. (1983). The interrater reliability of DSM-III in children. Journal of Abnormal Child Psychology, 11, 341-354.

Winer, B. J. (1971). Statistical procedures in experimental design. New York: McGraw-Hill.

Received October 30, 1989

Revision received March 22, 1990

Accepted April 2, 1990

\section{Butcher, Geen, Hulse, and Salthouse Appointed New Editors, 1992-1997}

The Publications and Communications Board of the American Psychological Association announces the appointments of James N. Butcher, University of Minnesota; Russell G. Geen, University of Missouri; Stewart H. Hulse, Johns Hopkins University; and Timothy Salthouse, Georgia Institute of Technology as editors of Psychological Assessment: A Journal of Consulting and Clinical Psychology, the Personality Processes and Individual Differences section of the Journal of Personality and Social Psychology, the Journal of Experimental Psychology: Animal Behavior Processes, and Psychology and Aging, respectively. As of January 1,1991, manuscripts should be directed as follows:

- For Psychological Assessment send manuscripts to James N. Butcher, Department of Psychology, Elliott Hall, University of Minnesota, 75 East River Road, Minneapolis, Minnesota 55455 .

- For JPSP: Personality send manuscripts to Russell G. Geen, Department of Psychology, University of Missouri, Columbia, Missouri 65211.

- For JEP: Animal send manuscripts to Stewart H. Hulse, Johns Hopkins University, Department of Psychology, Ames Hall, Baltimore, Maryland 21218.

- For Psychology and Aging send manuscripts to Timothy Salthouse, Georgia Institute of Technology, School of Psychology, Atlanta, Georgia 30332.

Manuscript submission patterns make the precise date of completion of 1991 volumes uncertain. Current editors will receive and consider manuscripts through December 1990. Should any 1991 volume be completed before that date, manuscripts will be redirected to the newly appointed editor-elect for consideration in the 1992 volume. 